\title{
Electrostatic solitary waves in current layers: from Cluster observations during a super-substorm to beam experiments at the LAPD
}

\author{
J. S. Pickett ${ }^{1}$, L.-J. Chen ${ }^{2}$, O. Santolík ${ }^{1,3,4}$, S. Grimald ${ }^{5}$, B. Lavraud ${ }^{6,7}$, O. P. Verkhoglyadova ${ }^{8,9}$, B. T. Tsurutani ${ }^{8}$, \\ B. Lefebvre ${ }^{2}$, A. Fazakerley ${ }^{5}$, G. S. Lakhina ${ }^{10}$, S. S. Ghosh $^{10}$, B. Grison ${ }^{3,11,12}$, P. M. E. Décréau ${ }^{13}$, D. A. Gurnett ${ }^{1}$, \\ R. Torbert ${ }^{2}$, N. Cornilleau-Wehrlin ${ }^{11,14}$, I. Dandouras ${ }^{6,7}$, and E. Lucek ${ }^{15}$ \\ ${ }^{1}$ Department of Physics and Astronomy, The University of Iowa, Iowa City, Iowa, USA \\ ${ }^{2}$ Space Science Center, University of New Hampshire, Durham, New Hampshire, USA \\ ${ }^{3}$ Institute of Atmospheric Physics, Prague, Czech Republic \\ ${ }^{4}$ Charles University, Faculty of Mathematics and Physics, Prague, Czech Republic \\ ${ }^{5}$ Mullard Space Science Laboratory, University College London, Holmbury St. Mary, UK \\ ${ }^{6}$ Centre d'Etude Spatiale des Rayonnements, Université de Toulouse (UPS), France \\ ${ }^{7}$ Centre National de la Recherche Scientifique, UMR 5187, Toulouse, France \\ ${ }^{8}$ Jet Propulsion Laboratory, California Inst. of Technology, Pasadena, California, USA \\ ${ }^{9}$ CSPAR, University of Alabama, Huntsville, Alabama, USA \\ ${ }^{10}$ Indian Institute of Geomagnetism, New Panvel (W), Navi Mumbai, India \\ ${ }^{11}$ LPP-CNRS, Vélizy, France \\ ${ }^{12}$ ESA/ESTEC, Noordwijk, The Netherlands \\ ${ }^{13}$ LPC2E, CNRS et Université d'Orléans, Orléans, France \\ ${ }^{14}$ Station de Radioastronomie de Nançay, Observatoire de Paris, CNRS, Nançay, France \\ ${ }^{15}$ The Blackett Laboratory, Imperial College, London, UK
}

Received: 28 February 2009 - Revised: 27 May 2009 - Accepted: 3 June 2009 - Published: 25 June 2009

\begin{abstract}
Electrostatic Solitary Waves (ESWs) have been observed by several spacecraft in the current layers of Earth's magnetosphere since 1982. ESWs are manifested as isolated pulses (one wave period) in the high time resolution waveform data obtained on these spacecraft. They are thus nonlinear structures generated out of nonlinear instabilities and processes. We report the first observations of ESWs associated with the onset of a super-substorm that occurred on 24 August 2005 while the Cluster spacecraft were located in the magnetotail at around 18-19 $R_{E}$ and moving northward from the plasma sheet to the lobes. These ESWs were detected in the waveform data of the WBD plasma wave receiver on three of the Cluster spacecraft. The majority of the ESWs were detected about 5 min after the super-substorm onset during which time 1) the PEACE electron instrument
\end{abstract}

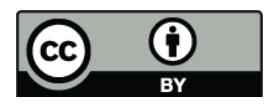

Correspondence to: J. S. Pickett (pickett@uiowa.edu) detected significant field-aligned electron fluxes from a few $100 \mathrm{eV}$ to $3.5 \mathrm{keV}$, 2) the EDI instrument detected bursts of field-aligned electron currents, 3) the FGM instrument detected substantial magnetic fluctuations and the presence of Alfvén waves, 4) the STAFF experiment detected broadband electric and magnetic waves, ion cyclotron waves and whistler mode waves, and 5) CIS detected nearly comparable densities of $\mathrm{H}+$ and $\mathrm{O}+$ ions and a large tailward $\mathrm{H}+$ velocity. We compare the characteristics of the ESWs observed during this event to those created in the laboratory at the University of California-Los Angeles Plasma Device (LAPD) with an electron beam. We find that the time durations of both space and LAPD ESWs are only slightly larger than the respective local electron plasma periods, indicating that electron, and not ion, dynamics are responsible for generation of the ESWs. We have discussed possible mechanisms for generating the ESWs in space, including the beam and kinetic Buneman type instabilities and the acoustic instabilities. Future studies will examine these mechanisms in more detail using

Published by Copernicus Publications on behalf of the European Geosciences Union and the American Geophysical Union. 
the space measurements as inputs to models, and better relate the ESW space measurements to the laboratory through PIC code models.

\section{Introduction}

Substorms at Earth (Akasofu, 1964) have been the subject of intense research for many years. Many aspects of substorms, such as what triggers their onset and where, and their significance in the global ongoing processes connected with the sun, have been studied in detail. Super-substorms are particularly intense substorms that show evidence of external triggering (Zhou and Tsurutani, 2001; Tsurutani and Zhou, 2003). We investigate a process involving nonlinear microphysics and the generation of Electrostatic Solitary Waves (ESWs) associated with the onset of a super-substorm that occurred on 24 August 2005 during the main phase of a severe magnetic storm. The purpose of the present study is not to investigate in detail the super-substorm itself, but rather to discuss the effects of that super-substorm on physical processes in the magnetotail.

In the present study we use data from the four Cluster spacecraft, which were located in the magnetotail at 18-19 $R_{E}, \sim 01: 00$ Magnetic Local Time and -10 to -12 degrees Solar Magnetic latitude, to investigate the physical processes observed at Cluster's location in association with the supersubstorm onset. We analyzed data from several of the Cluster instruments, namely: Wideband Data (WBD) plasma wave receiver (Gurnett et al., 1997), Electron Drift Instrument (EDI) (Paschmann et al., 1997), Flux Gate Magnetometer (FGM) (Balogh et al., 1997), Spatio-Temporal Analysis of Field Fluctuations Search Coil (STAFF-SC) and Spectrum Analyzer (STAFF-SA) (Cornilleau-Wehrlin et al., 2003), Electric Field and Wave Experiment (EFW) (Gustafsson et al., 1997), WHISPER Resonance Sounder (Décréau et al., 1997), Plasma Electron and Current Experiment (PEACE) (Johnstone et al., 1997), and Cluster Ion Spectrometry (CIS) (Rème et al., 2001). The primary focus of this investigation is nonlinear Electrostatic Solitary Waves (ESWs) observed shortly after super-substorm onset, with comparison to ESWs observed in recently-conducted laboratory experiments at the University of California-Los Angeles plasma device (LAPD).

ESWs are small-scale structures representing 1) holes in either electron or ion phase space or 2) density enhancements and decreases, depending on whether they are BernsteinGreene-Kruskal (BGK) structures generated out of: 1) a beam, two-stream, or kinetic Buneman type of instability (Bernstein et al., 1957; Omura et al., 1996; Goldman et al., 2003; Chen et al., 2004, 2005) or 2) an acoustic mode generated out of an acoustic instability (Dubouloz, 1991; Berthomier et al., 2000; Singh and Lakhina, 2001; Singh et al., 2001; Kakad et al., 2007; Ghosh et al., 2008; Lakhina et al., 2008a, b), respectively. ESWs have been observed in the magnetotail by a few missions, most notably and first by Geotail (Matsumoto et al., 1994) in the far tail, followed by Polar (Franz et al., 1998, 2000; Cattell et al., 1999) in the near Earth plasma sheet and by Cluster in the mid-tail plasma sheet (Pickett et al., 2004). They are observed in the data as isolated solitary pulses or sometimes as a series of several pulses (see Fig. 3). They have also been observed in association with reconnection in the magnetotail (Cattell et al., 2005; Deng et al., 2006) and with dayside reconnection through observations in the cleft (Pottelette and Treumann, 1998) and in the magnetopause boundary (Matsumoto et al., 2003). A statistical study by Pickett et al. (2004) showed that ESWs are observed throughout much of the Cluster orbit ( $\sim 4 \times 19 R_{E}$ during the first few years of the mission), but are primarily confined to boundary or current layers or wherever turbulence is present, such as throughout the magnetosheath (Pickett et al., 2005). Their presence thus indicates a plasma that is unsettled with at least one or more active instabilities involving trapped particles or density perturbations.

In Sect. 2, we present the Cluster observations that are relevant to the study of the ESWs associated with this supersubstorm. The Cluster wave observations of the ESWs are discussed first, followed by the supporting data from the other Cluster instruments that help us understand the plasma in which the measurements were made. Section 3 is dedicated to a discussion of ongoing laboratory experiments being carried out in support of studies of ESWs in space, including a comparison of the ESWs observed in space to those observed in the LAPD. In Sect. 4 we briefly discuss the possible ESW generation mechanisms for the ESWs observed during the LAPD experiments and during the super-substorm based on the observations. In Sect. 5 we present our conclusions.

\section{Space observations}

As determined by the IMAGE spacecraft Wideband Imaging Camera (WIC) instrument, onset of the super-substorm at Earth was around 09:42 UT on 24 August 2005. The auroral bulge phase of the substorm around 09:48 UT is the phase that is relevant to our study of ESWs. For more information about this super-substorm and analysis of it, the reader is referred to the first Virtual Conference website (http://workshops.jhuapl.edu/), hosted by Johns Hopkins University in November 2006.

\subsection{ESW data}

Figure 1 shows the observations of WBD on Cluster spacecraft (SC) 1, 2 and 4 in the time period 09:00 to 11:00 UT. Data from SC3 were not obtained in this interval by WBD. However, SC3 was close to SC4 and would have seen essentially the same overall wave profile as SC4. At this time the 


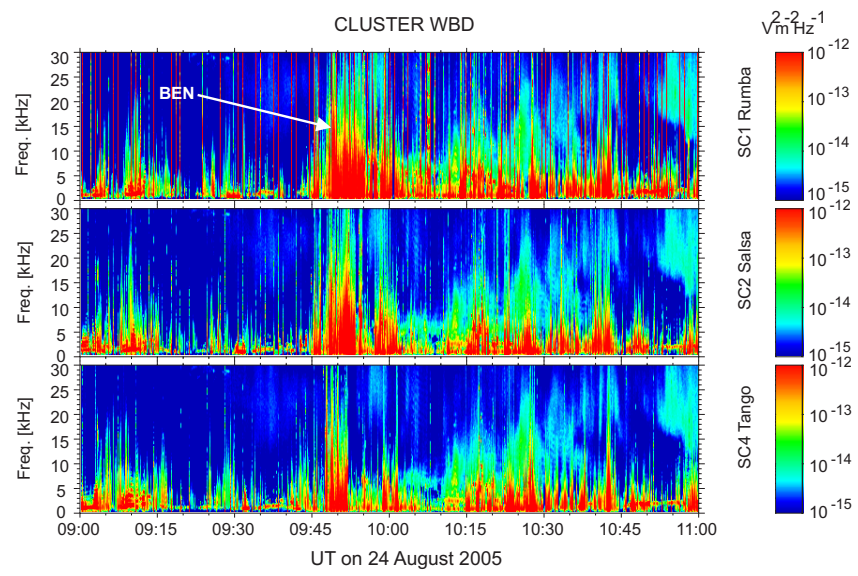

Fig. 1. Cluster WBD data during the 24 August 2005 supersubstorm. Shown are data from three of the Cluster spacecraft, SC1, SC2 and SC3, from top to bottom, for the time period 09:00 to $11: 00 \mathrm{UT}$, frequency range of $100 \mathrm{~Hz}$ to $30 \mathrm{kHz}$, with color indicating electric field power spectral density. Of interest is the BEN seen on all three spacecraft beginning around 09:45-09:48, which is a few minutes after the onset of the super-substorm.

spacecraft were configured as shown in Fig. 2 in the GSE X$\mathrm{Z}$ and $\mathrm{X}-\mathrm{Y}$ planes, with the spacecraft moving northward with increasing time from the plasma sheet to the lobe and back to the plasma sheet. SC4 was closest to Earth, followed by SC3, SC1, and SC2 (farthest). In terms of Geomagnetic Latitude, SC3 was at the highest latitude, followed by SC4, SC2 and SC1. The inter-spacecraft distances were: 4-3: $830 \mathrm{~km}, 3-1: 6724 \mathrm{~km}, 1-2: 8359 \mathrm{~km}$. Even at these large spacecraft distances, the waves detected on all Cluster spacecraft are very similar with differences being noted primarily in UT time of observation and duration of detection.

Looking again at Fig. 1, we point out the broadband waves designated as BEN (Broadband Electrostatic Noise) that extend from the lowest frequencies measured $(\sim 100 \mathrm{~Hz})$ up to $20-30 \mathrm{kHz}$. These are the waves that are the focus of this study. Around 09:45 UT, the first disturbance associated with the super-substorm is observed about $3 \mathrm{~min}$ after onset. An analysis of the high time resolution WBD data shows that the disturbance is seen first on SC2, followed by SC1, then SC4. Since SC2 is the farthest from the Earth, it is a disturbance initiated even farther from Earth and beyond SC2's location, $18.9 R_{E}$. The main disturbance is then observed around 09:47:30 UT in association with the auroral bulge phase of the super-substorm. The broadband waves are observed extending to higher frequencies (implying shorter time scale) and are very intense. In this case, the order in which the spacecraft observe the disturbance ( $\mathrm{SC} 4, \mathrm{SC} 1, \mathrm{SC} 2)$ is exactly reversed from the previous one at 09:45 UT, implying that the disturbance is taking place closer to Earth than the location of the Cluster spacecraft and propagating outward down the magnetotail. Furthermore, this disturbance, with the associated broadband waves, persists throughout the re-
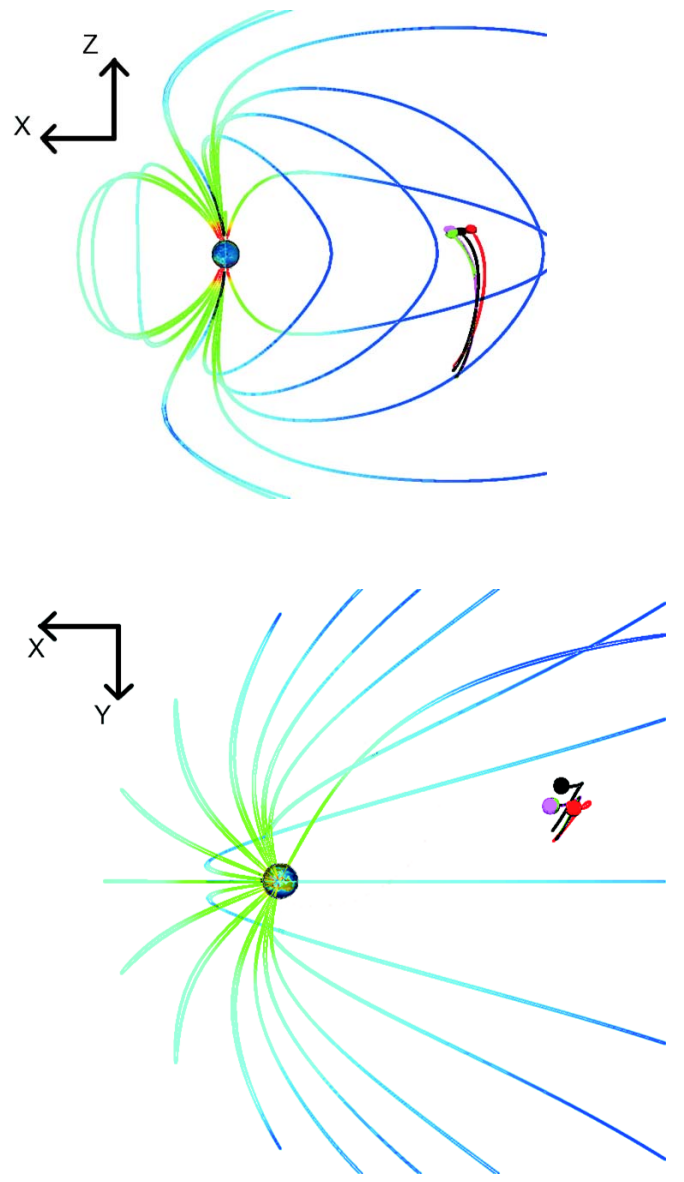

Fig. 2. Location of the four Cluster spacecraft in the $X_{\mathrm{GSE}}-Z_{\mathrm{GSE}}$ (top) and $X_{\mathrm{GSE}}-Y_{\mathrm{GSE}}$ (bottom) coordinate planes at the time of the super-substorm. The four Cluster satellites are located in the magnetotail around $18-19 R_{E}$ and are color coded as follows: $\mathrm{SC} 1$ : black, SC2: red; SC3: green; SC4: magenta. SC3 and SC4 are closest to Earth; SC2 is farthest. The spacecraft are moving northward with increasing time from the plasma sheet to the lobe.

mainder of the time period in which WBD data were obtained through about 12:15 UT. However, the frequency extent of the broadband waves after about 09:52 to 09:55 UT is significantly reduced.

In order to understand the makeup of the broadband waves, we show a $2.5 \mathrm{~ms}$ length time vs. electric field amplitude plot of the waveforms at 09:48:36 in the top panel of Fig 3. These waveforms, when analyzed with a Fast Fourier Transform (FFT), result in the broadband waves observed in Fig. 1. In Fig. 3 we observe four major pulses all with time durations of about $214 \mu \mathrm{s}$ and amplitudes of a few $\mathrm{mV} / \mathrm{m}$, with ESW spacing about $270 \mu \mathrm{s}$. In comparison, the electron plasma period at a density of $\sim 1.5 \mathrm{~cm}^{-3}$ is about $90 \mu$ s based on Cluster EFW spacecraft potential and Whisper sounder data. These coherent, isolated pulses observed in the high time resolution AC electric field waveform data are referred to as ESWs (Matsumoto et al., 1994). Small amplitude solitary 

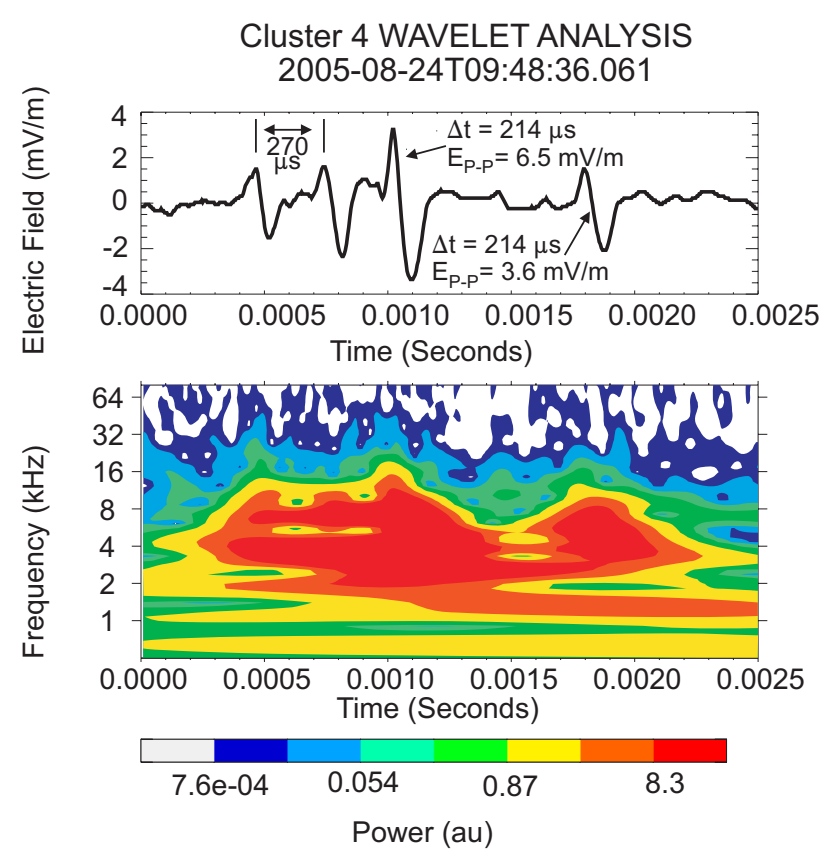

Fig. 3. Cluster WBD data taken on SC4 during the time of greatest disturbance at Cluster's location at 09:48:36. Top panel: waveform data recorded over a $2.5 \mathrm{~ms}$ time interval showing some of the ESWs observed during this time, with their noted characteristics defined by pulse duration and electric field amplitude. Bottom panel: wavelet transform of the data shown in the top panel plotted as frequency vs. time with color indicating the power of the waves in arbitrary units (au). The ESWs appear as localized structures around 3 to $8 \mathrm{kHz}$.

waves containing magnetic-field aligned electric field components were first observed in space on the S3-3 spacecraft (Temerin et al., 1982) in the auroral region, but these solitary waves were not identified as to the type of structure they represented (i.e., phase space holes or density enhancements or depletions).

A wavelet transform, using a Morlet reference wavelet, of the waveforms shown in the top panel of Fig. 3 results in the wavelet spectrogram seen in the bottom panel of Fig. 3. The wavelet transform highlights the true nature of the ESWs, i.e., they are localized in time and frequency. The characteristic frequencies of about 3 to $12 \mathrm{kHz}$ represent the ESW pulse durations, as well as the time between individual pulses. For example, the inverse of the average of this frequency range gives a characteristic time of 333 to $83 \mu \mathrm{s}$, which is consistent with the time durations of the ESWs, as well as the time between successive ESWs, shown at the top of Fig. 3. Referring back to Fig. 1, we can now say that the ESWs observed around 09:47:30 UT to 09:55:00 UT, i.e., the auroral bulge phase, have time durations substantially shorter (typically few $100 \mu$ s but can be as low as about $70 \mu \mathrm{s}$ ), for the most part, than those observed both before and after this time period, which are on the order of $1 \mathrm{~ms}$ (not shown here).
The shorter ESW time durations observed in the bulge phase are most likely due to coincident enhanced field-aligned (in both parallel and anti-parallel directions, consistent with the mixed polarity of the ESWs - see next paragraph) electron fluxes, as shown in the first and third panels of Fig. 7 and as discussed in Sect. 2.2, as opposed to increased plasma density. The plasma density in the bulge phase is lower than that before the bulge phase and higher than that after (the bulge phase is a transition stage), according to the density measurements from CIS (see Panels 1 and 2 of Fig. 6), EFW (deduced from the spacecraft potential measurements), and Whisper sounder measurements. ESWs with longer time durations around $1 \mathrm{~ms}$ are more common in the magnetotail (see Pickett et al., 2004), implying that the physical processes associated with the major super-substorm disturbance at Cluster around 09:47-09:55 are altered as compared to more quiet times in the plasma sheet.

Finally, we mention for completeness that Cluster WBD is unable to distinguish positive potential polarity ESWs from negative ones or obtain propagation speed on any one single Cluster spacecraft because it simply measures the potential difference between two electric field spheres (a dipole) along only one axis. With regard to whether all detected ESWs for this event are of the same electric-field polarity, they are not. In fact in intervals as small as a few ms we can see ESWs with opposite electric-field polarities (referred to the first excursion of the bipolar pulses) which cannot be attributed to the spinning antenna's orientation with respect to the ESWs' propagation. However, we do not know whether these are ESWs of the same potential polarity traveling in opposite directions, or whether they are opposite potential polarities traveling in the same direction. If the ESWs are of similar time duration as is usually the case, we would tend to favor the former explanation, and this is consistent with the enhanced directional fluxes of electrons both parallel and antiparallel to the magnetic field (see Fig. 7). However, we stress that there is no way to prove this with the WBD data alone.

We have been able to obtain propagation speed in a very limited number of cases in the auroral zone (Pickett et al., 2004) and at the magnetopause boundary layer (Pickett et al., 2008) when the Cluster spacecraft were configured with mean separation distances of $200 \mathrm{~km}$ or less. As noted above, for this super-substorm event the spacecraft were separated on the order of 1000 to $8000 \mathrm{~km}$, making it impossible to observe propagation of one ESW from one spacecraft to another. In Polar observations of ESWs at the plasma sheet boundary layer (which is the relevant region to this study), the ESWs propagate typically at around the electron thermal speed or higher (Franz et al., 2005). Even though Cluster WBD cannot measure the speeds of ESWs for the super-substorm event, the fact that the measured ESWs have time durations comparable to the electron plasma period suggests that they might propagate at the electron thermal speed. WBD is also not able to provide direction of ESW propagation, i.e., parallel, oblique, perpendicular, or anti-parallel to 
the magnetic field since its measurements are made along only one axis, that being in the spin plane. However, we are able to transform the three-axis FGM magnetic field data into the antenna coordinate system used by WBD in order to obtain the angle between the WBD measurement antenna and the magnetic field $\left(\theta_{\text {Ant-B }}\right)$. If the ESW structures are propagating parallel or anti-parallel to the magnetic field, a necessary condition for this to be the case is that $\theta_{\text {Ant-B }}$ must preferably be near 0 or 180 degrees, or at least not close to 90 degrees. This is indeed the case for all of the ESWs observed during and following the super-substorm onset, with the occurrence probability of $\theta_{\text {Ant-B }}$ strongly peaking at 0 and 180 degrees.

\subsection{Supporting data}

In order to put the ESW measurements into context with the plasma environment in which they are observed, we next analyze other Cluster data taken in situ. Figure 4 contains EDI and FGM data from SC1 covering the interval 09:40 to 10:05 UT on 24 August 2005. The top two panels contain electron fluxes (in units of $\left(1 /\left(\mathrm{cm}^{2}-\mathrm{sec}-\mathrm{sr}-\mathrm{eV}\right)\right)$ deduced from the EDI measurements of $500 \mathrm{eV}$ electrons at 0-degree and 180-degree pitch angles, respectively. Note the different amplitude scales on these two panels. Of primary significance is the occurrence of simultaneous fluxes of different magnitudes at pitch angles 0 and 180 degrees, implying the presence of field-aligned electron currents. As mentioned in the Introduction, ESWs are usually found in all boundary and current layers.

The FGM DC magnetic field measurements of the $B x$ (Blue), $B y$ (green) and $B z$ (red) components of the magnetic field in a GSE coordinate system are shown in the third panel. These measurements show large fluctuations in the magnetic field beginning around 09:45 UT and continuing throughout the 25-min period, but most strongly in the interval of 09:47 to 09:52 UT where the bulk of the ESWs are observed (bottom panel). These fluctuations in the magnetic components usually indicate the presence of currents. We also note that $B x$ is primarily negative during this interval. The total magnetic field (black line) and detections of ESWs by WBD (black and green dots) are shown in the bottom panel. The ESWs are automatically detected by an analysis routine specifically designed to identify isolated bipolar pulses (two humps similar to those shown in Fig. 3) and tripolar pulses (three humps, with the third hump having the same sign as the first) in the waveform data (see Pickett et al., 2004). In the bottom panel of Fig. 4, black dots represent detections of bipolar pulses and green dots represent tripolar pulses. The amplitude of these pulses, in $\mathrm{mV} / \mathrm{m}$, is obtained by referring to the left vertical axis, while the magnetic field amplitude scale is given on the right vertical axis. This panel shows that most ESWs have amplitudes of 0.1 to $10 \mathrm{mV} / \mathrm{m}$ and are detected in regions where currents are present and the magnetic field fluctuations are largest.
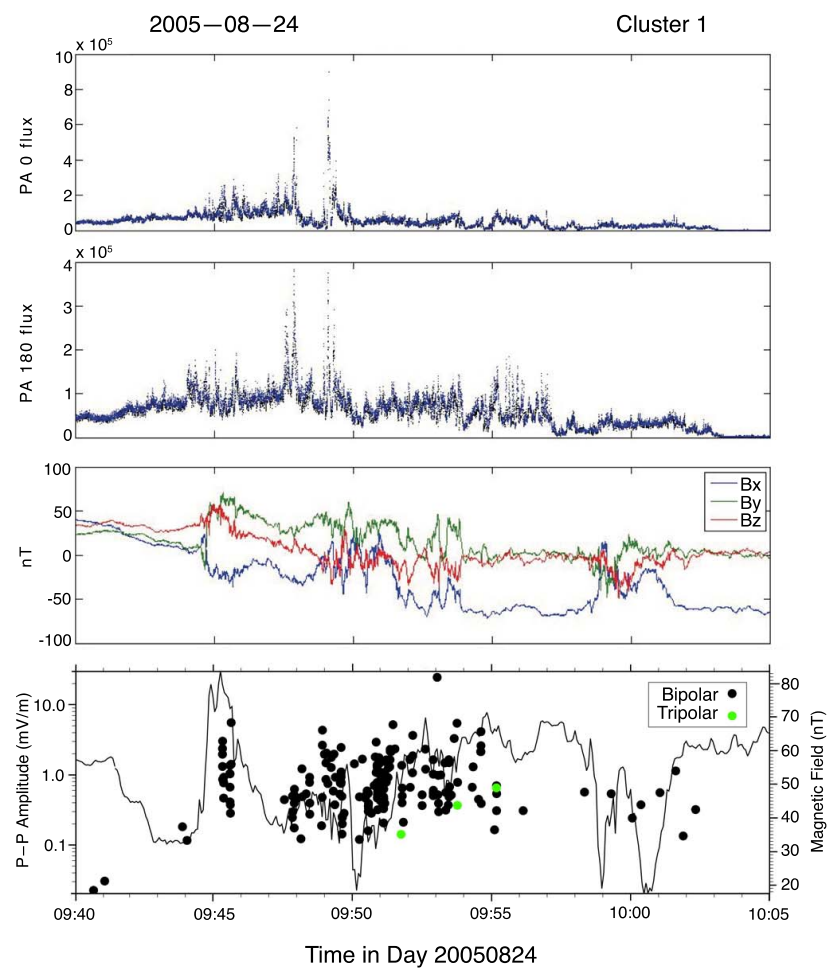

Fig. 4. EDI, FGM and WBD data obtained on Cluster SC1 during the period of the most intense disturbance from 09:45 to 09:55. Top two panels: magnetic field aligned and anti-aligned fluxes of electrons as deduced from the EDI measurements of 500-eV ambient electrons. Third panel: The $B x, B y$, and $B z$ components of the magnetic field as measured by FGM, showing intense fluctuations in the period of 09:45 to 09:55. Bottom panel: analyzed WBD data showing when bipolar (black dots) and tripolar (green dots) ESWs are detected and their electric field amplitudes given on the left horizontal axis. The total magnetic field is plotted as a solid black line with the scale on the right vertical axis. Most ESWs are detected during the intense magnetic fluctuations when currents are observed.

Next we look at some of these magnetic fluctuations in more detail. The hodogram plotted in Fig. 5 shows the B1 vs. B2 components obtained through Minimum Variance Analysis (MVA) of the magnetic field data (Sonnerup and Cahill, 1967) for the time interval 09:48:50.4 to 9.49:26.4 UT at the height of the disturbance at SC1. In the MVA coordinate system, B1, B2 and B3 are the field components in the maximum, intermediate and minimum variance directions. The hodogram shows that most of the perturbation field is in the B1 direction, suggesting the presence of linear polarized Alfvén waves. Several other intervals were analyzed with MVA and found to contain similar signatures of linearity, suggesting that Alfvén waves are present throughout much of the major disturbance interval of 09:47 to 09:55 UT. 
Cluster 1 FGM Data

24 August 2005, 09:48:50.4 to 09:49:26.4 UT

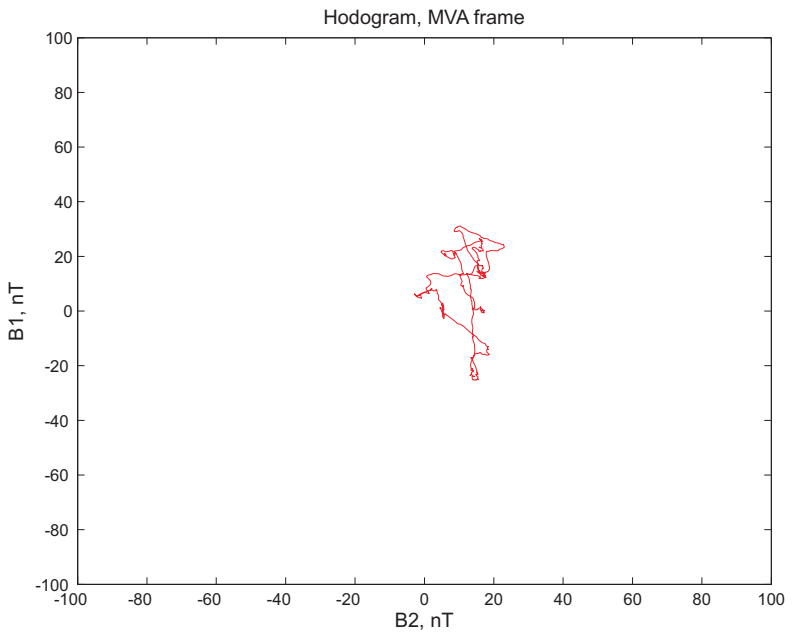

Fig. 5. Minimum variance analysis of an approximate $30 \mathrm{~s}$ interval of magnetic field data obtained during the time when most ESWs are observed. The hodogram of the B1 vs. B2 components in the MVA frame show that the Alfvén wave is linearly polarized.

The CIS CODIF ion data and STAFF-SC low frequency magnetic field data for the time period 09:35 to 10:05 UT on SC4 are presented in Fig. 6. The top two panels show the $\mathrm{H}+$ and $\mathrm{O}+$ densities, respectively, in $\mathrm{cm}^{-3}$. The next two panels show the $\mathrm{H}+$ and $\mathrm{O}+$ parallel and perpendicular temperatures, respectively, in $\mathrm{eV}$. The fifth panel provides the $\mathrm{H}+$ velocity over the range of $\sim 30 \mathrm{eV}$ to $40 \mathrm{keV}$. We note that there is a small but significant $\mathrm{H}+$ and $\mathrm{O}+$ temperature anisotropy (Tpar $>$ Tper) in the period where most of the ESWs are detected on this spacecraft (i.e., 09:47 to $09: 53 \mathrm{UT}$ ) and that the $\mathrm{H}+$ velocity in this time period is steadily increasing in the tailward direction to a rather high velocity of $\sim 700 \mathrm{~km} / \mathrm{s}$ at 09:51:30 UT. At this point the sign of the ion velocity reverses and the ESWs (see broadband waves in Fig. 1, SC4) disappear. Although the overall density is low (around $1.5 \mathrm{~cm}^{-3}$ ) in this time interval, the $\mathrm{O}+$ density in the time of increasing tailward flow becomes a substantial part of the total density (about 0.2 vs. 0.4 for $\mathrm{H}+$ ). Note that around the end time of the plot, where the density is lowest, the CIS CODIF data are not valid owing to the spacecraft entering into the lobes (empty region with low count rates). Based on these ion data and the data shown in Fig. 4, we have a current layer in which two ion species are dominant, as opposed to one. Corresponding precisely with the increasing tailward flow are broadband electromagnetic waves in the frequency range of about $0.3 \mathrm{~Hz}$ to $12 \mathrm{~Hz}$ as shown by the STAFF-SC data plotted in the bottom panel of Fig. 6. Plotted is the sum of all three orthogonal magnetic components of the waves over the stated frequency range with color indicating magnetic field intensity. Analysis of these waves shows that ion cyclotron waves around $0.6 \mathrm{~Hz}$ are likely present (not shown).

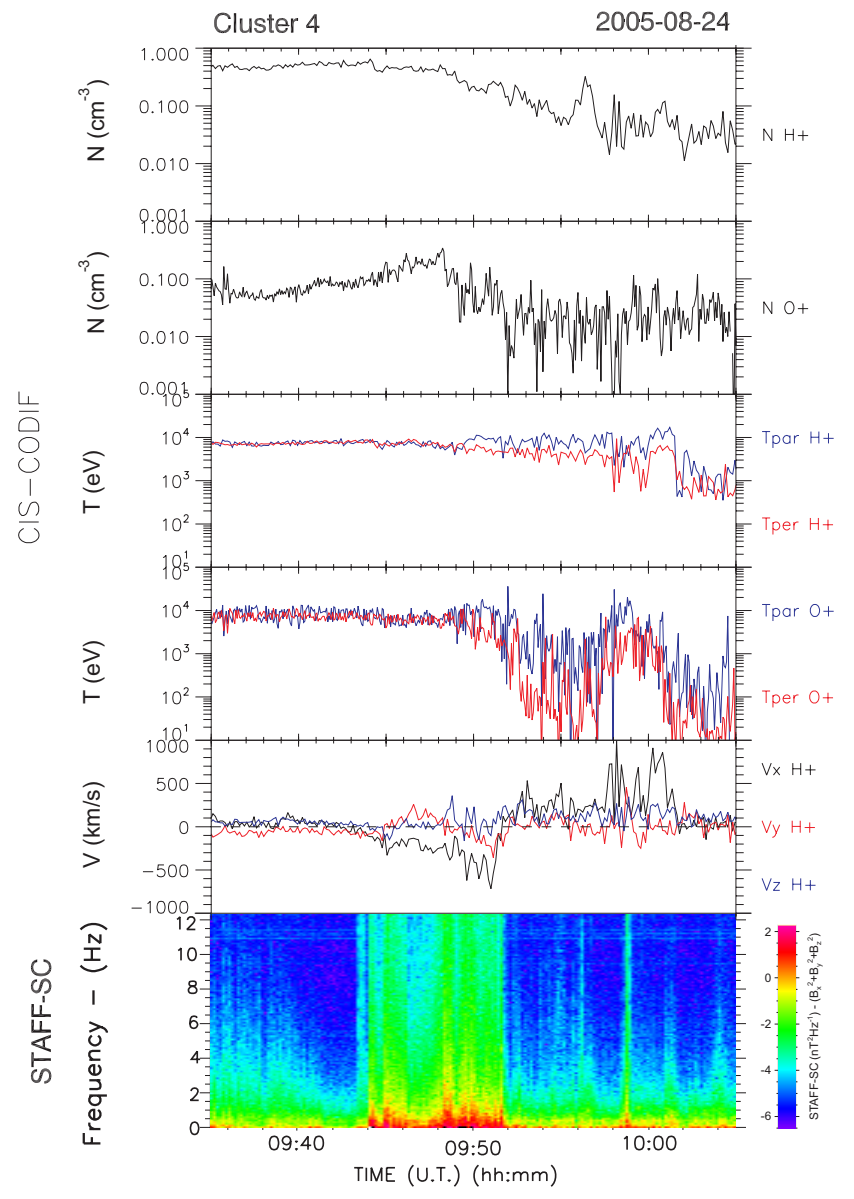

Fig. 6. Cluster SC4 data from the CIS CODIF and STAFF-SC instruments. Top two panels: density of $\mathrm{H}+$ and $\mathrm{O}+$, respectively: panels three and four: parallel and perpendicular $\mathrm{H}+$ and $\mathrm{O}+$ temperatures, respectively. These show a small amount of anisotropy during the period of interest for ESWs, i.e., 09:47-09:53. Panel five: $X, Y$ and $Z$ components of the $\mathrm{H}+$ velocity in the GSE coordinate system, showing an increasing negative $X$ velocity from 09:44 to 09:52. Bottom panel: magnetic field power spectral density of the waves as the sum of all three orthogonal components. Broadband waves in the frequency range $\sim 0$ to $12 \mathrm{~Hz}$ are observed during the time when the ion velocity is steadily increasing in the negative $X_{\mathrm{GSE}}$ direction.

With regard to the electron data obtained by the PEACE instrument, up to about 09:44 UT there is some heating of the electrons which is normal for this region. At this time the distribution is also isotropic in the range of about $160 \mathrm{eV}$ to $13 \mathrm{keV}$. In the period of 09:44 to 09:54 UT there is a small increase in flux observed around 09:44:30 UT with fieldaligned fluxes observed shortly after. Then, more intense field-aligned and counter-streaming fluxes dominate for the interval 09:48 to 09:54 UT as shown in the top three panels (parallel, perpendicular and anti-parallel to the magnetic field, respectively) of Fig. 7. In these panels the electron energy is plotted vs. time for SC1 with the color indicating the 


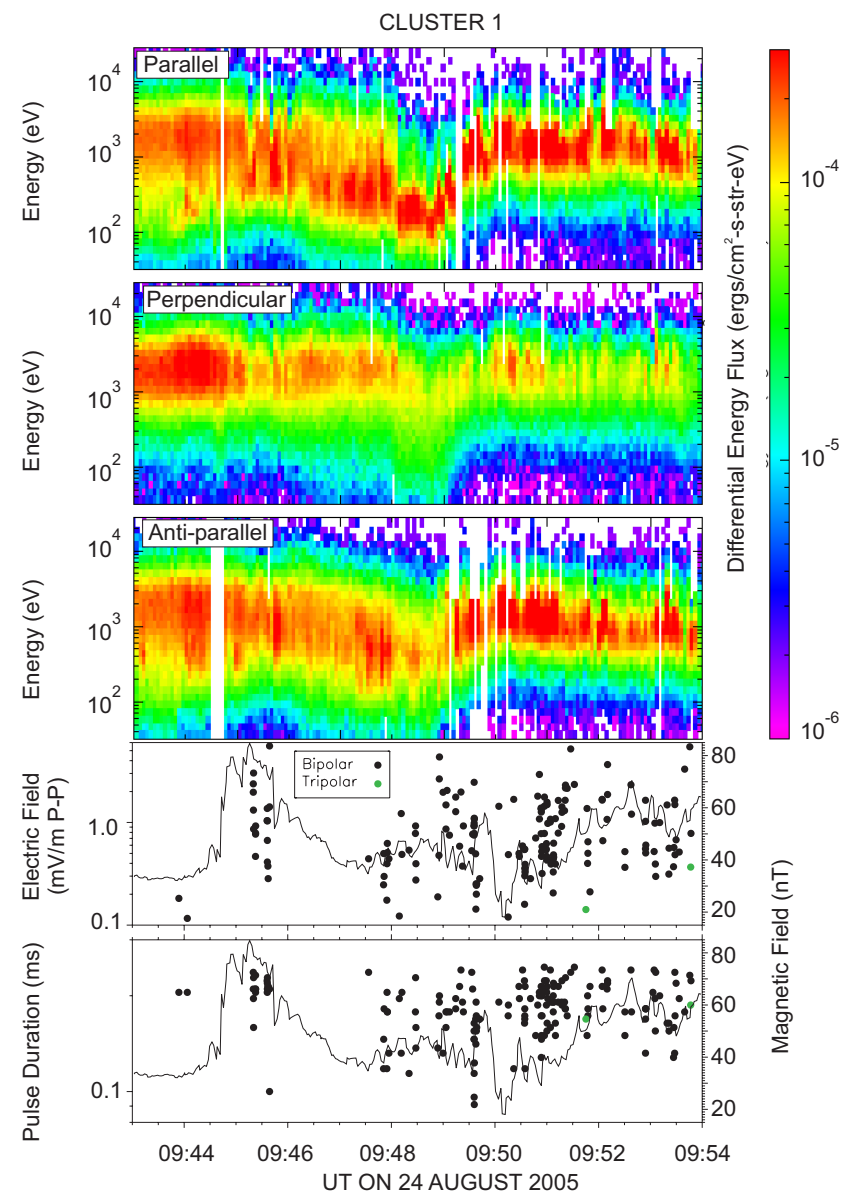

Fig. 7. PEACE and WBD ESW data on Cluster SC1. Top three panels: electron energy vs. time with color indicating the differential energy flux for fluxes parallel, perpendicular and anti-parallel to the magnetic field, respectively. Field-aligned fluxes are clearly seen in the interval 09:48 to 09:53. Bottom two panels: the first panel shows the ESW detections with format the same as in the bottom panel of Fig. 4. The bottom panel of Fig. 7 has the same format except that the time duration of the ESWs is plotted on the left vertical axis. This figure shows clearly that the ESWs are most strongly detected in the regions of field-aligned fluxes.

differential energy flux. The field-aligned fluxes from 09:48 to 09:54 UT are primarily in the energy range of $380 \mathrm{eV}$ up to $3.5 \mathrm{keV}$, but are slightly lower in energy at the start of the interval. These field-aligned fluxes are not highly beamed during this interval but rather distributed over a slightly broader energy range. The detections of the ESWs by the WBD instrument for SC1 for the period 09:43 to 09:54 UT are shown at the bottom of Fig. 7. The format is the same as that shown at the bottom of Fig. 4 except that the second WBD panel shows the time duration of each detected bipolar and tripolar pulse. From these WBD data we find that the ESWs are observed predominantly in the period 09:48 to 09:54 UT with pulse time durations on the order of 80 to $250 \mu$ s and amplitudes ranging from 0.1 to $10 \mathrm{mV} / \mathrm{m}$. Thus, Figs. 6 and 7 show

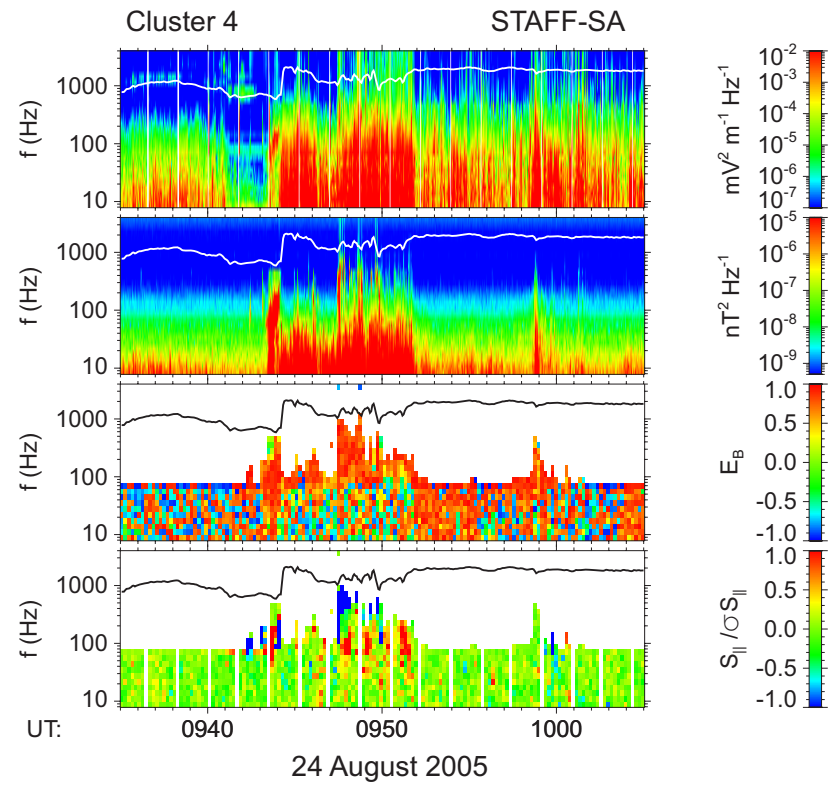

Fig. 8. STAFF-SA data for the period of 09:35-10:05 on SC4. Top two panels: sum of the power spectral densities of the two electric components and the three magnetic components, respectively. Broadband waves up to about $100 \mathrm{~Hz}$ and higher frequency bursts around a few $100 \mathrm{~Hz}$ are visible in the primary disturbance period of 09:44 to 09:53. Third panel: elipticity of the waves, showing that the higher frequency bursts are nearly circular. Bottom panel: $Z$ component of the Poynting flux showing that the higher frequency bursts, which are less than the electron cyclotron frequency, are propagating in both directions. Based on these data, the higher frequency bursts are whistler mode waves.

us that there is a clear association of detection of the ESWs during the time of intense field-aligned electron fluxes, as well as with detection of the tailward flow of ions where the $\mathrm{H}+$ and $\mathrm{O}+$ densities are of the same order of magnitude. As previously mentioned, the ESWs are also detected when the electric field antenna used to make the measurements is nearly aligned or anti-aligned with the magnetic field, suggesting that observed field-aligned electron fluxes could be important in the generation process of the ESWs.

To conclude our discussion of supporting data, we present in Fig. 8 the STAFF-SA data taken during the period 09:35 to $10: 05$ on SC4 in the frequency range $10-4000 \mathrm{~Hz}$. The top two panels provide the power spectral density of the electric and magnetic components, respectively, the third panel shows the ellipticity of polarization and the bottom panel shows the Z-component of the Poynting vector, with the black/white line being the electron cyclotron frequency. We see intense broadband electric and magnetic waves up to about $100 \mathrm{~Hz}$, and higher frequency bursts (few hundred $\mathrm{Hz}$ ) in the period 09:44 to 09:53. The high frequency bursts appear to be circularly polarized (third panel, ellipticity close to 1) at frequencies less than the electron cyclotron frequency, thus whistler mode. They also appear to be propagating both 


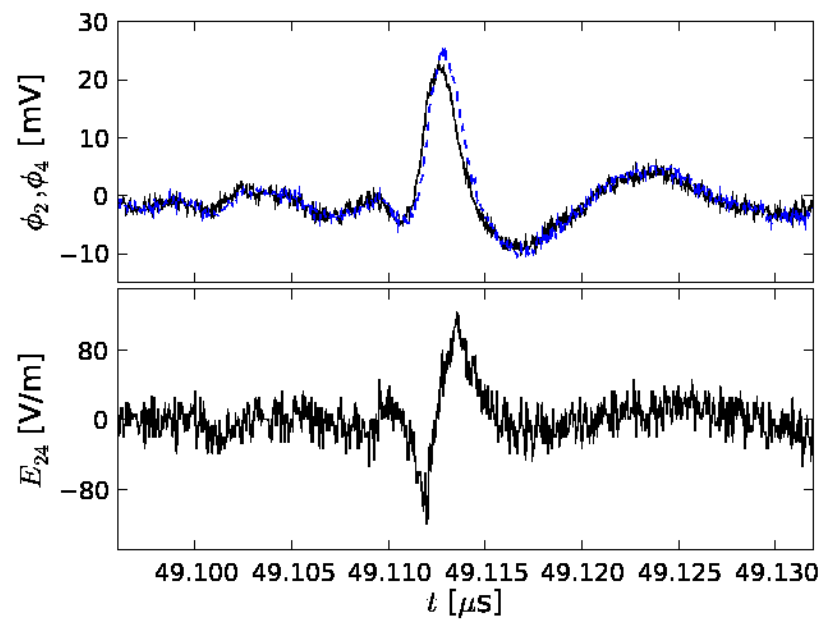

Fig. 9. Example of an ESW produced in the laboratory using the LAPD with an injected beam. Top panel: potentials as measured by two different probes separated by $80 \mu \mathrm{m}$ with probe 2 (black) detecting the ESW slightly earlier than Probe 4 (blue). Bottom panel: the electric field calculated as the difference of the two potentials divided by the probe separation.

along and opposed to the magnetic field direction (bottom panel, Z-component of Poynting vector close to 1 and -1 , respectively). The high frequency bursts appear to be associated with the time period of field-aligned fluxes as observed in the electron data presented in Fig. 7.

\section{Laboratory experiments}

In order to gain some insight into the space observations we have begun to run a series of experiments in the University of California-Los Angeles plasma device (LAPD) to generate ESWs and to study their roles in thin current layers. For a description of the LAPD and the research conducted with this facility, refer to the website http://plasma.physics.ucla. edu/bapsf/index.html. For our first experiments we injected an electron beam into a cold plasma and varied the speed of the beam, as well as the magnetic field strength. We also took measurements in a wide range of background plasma densities. The first of these results will be published elsewhere by Lefebvre et al. (2009) Here we present an example of the ESWs to demonstrate that we have been able to generate them in the laboratory by injecting an electron beam, and to detect them at locations 500-1000 $\lambda_{D e}$ (Debye lengths) from the beam source.

Figure 9 shows an example of a solitary wave that was generated in the LAPD with a $90 \mathrm{eV}$ electron beam. The magnetic field strength was set to 750 Gauss, the electron temperature was $0.2 \mathrm{eV}$, and the plasma density was $4 \times 10^{9} \mathrm{~cm}^{-3}$. The plasma can be considered collisionless at the experiment's scale, because the electron-electron collisional mean free path is about $17 \mathrm{~cm}$, while the measurements were taken at about $6 \mathrm{~cm}$ from the beam source. In the top panel are plotted the potential measurements made by Probes 2 (black) and 4 (blue) which were $80 \mu \mathrm{m}$ apart. The electric field obtained from these measurements (difference between the two probe potentials divided by their separation) resulted in the trace seen in the bottom panel. This figure demonstrates that we have generated a solitary wave with electric field of $240 \mathrm{~V} / \mathrm{m}$ peak-to-peak and time duration of $0.0029 \mu \mathrm{s}$ (slightly larger than one electron plasma period: $f_{p e}^{-1}=0.0025 \mu \mathrm{s}$ ). Based on the measured time delay from probe 2 to probe 4 of $0.0002 \mu \mathrm{s}$ and the distance traveled in that time (probe distance of $80 \mu \mathrm{m}$ ), we have calculated that this ESW travels away from the beam source at a speed of $300 \mathrm{~km} / \mathrm{s}$ (parallel to the background magnetic field), equivalent to $1.21 v_{T e}$ (thermal speed of background electrons) and $0.053 v_{b}$ (beam speed), and has a size of $8.8 \lambda_{D e}\left(1 \lambda_{D e}\right.$ is about $56 \mu \mathrm{m}$ for the density and temperature in this particular run). The fact that the ESW velocity is so much smaller than the beam speed rules out the electron-electron two stream instability as the generation mechanism for the LAPD ESWs because the two-stream generated ESWs are expected to propagate at about half the beam speed. In addition, we can rule out the possibility that the LAPD ESWs are ion acoustic mode since they propagate at a few hundred $\mathrm{km} / \mathrm{s}$, whereas the ion acoustic speed is a few $\mathrm{km} / \mathrm{s}$.

The ESW scales in the space observations discussed in Sect. 2 are very different from those in the laboratory due to the disparate densities and temperatures in space and the laboratory. For the super-substorm event, the ESWs were on the order of $200 \mu \mathrm{s}$ time duration $\left(f_{p e}^{-1} \sim 90 \mu \mathrm{s}\right)$, or about 70000 times longer than the laboratory, and $0.004 \mathrm{~V} / \mathrm{m}$ amplitude, or about 60000 times smaller than the laboratory. However, both time durations of the space and laboratory ESWs are close to, but slightly larger than, the electron plasma period, suggesting that they are both electron-mode solitary waves. It is not possible to obtain the velocity and size of the ESWs for this super-substorm case as discussed at the end of Sect. 2.1. Since it is also impossible to exactly recreate the space plasma in the laboratory, we must go one step further to deal with these vastly different scales. In future studies we will 1) simulate the laboratory parameters with 2-D and 3-D PIC code; 2) make systematic comparisons between laboratory and simulation results to establish correspondence; and 3) vary the parameters in PIC simulations to study the cases from space. This should provide us with a better understanding of the measurements made in space since both the space and laboratory measurements are being made within current layers with measured characteristics.

\section{Discussion of ESW generation mechanisms}

Many papers have been written over the last few years which attempt to determine how the ESWs observed in space are generated (see Introduction to Franz et al. (2005) for a re- 
cent review). Many of them are purely observational using space observations, many others are purely theoretical with or without simulations, and others combine observations and theory. Most attempt to explain the generation of these ESWs through beam, counter-streaming or kinetic Buneman instabilities (Omura et al., 1996; Goldman et al., 2003; Chen et al., 2004, 2005), whether with electrons or ions, or both, involved, and as a product of magnetic reconnection (Pottelette and Treumann, 1998; Matsumoto et al., 2003; Drake et al., 2003; Cattell et al., 2005). The ESWs that result from these types of instabilities represent electron or ion holes in phase space and are known as BGK type structures (Bernstein et al., 1957). In addition, there has been an attempt to explain the ESWs as density structures (enhancements or decreases) which result from ion and electron acoustic instabilities (e.g., Berthomier et al., 2000; Singh et al., 2001; Ghosh et al., 2008; Lakhina et al., 2008a, b). Although all of the proposed explanations appear plausible based on the observations and models/simulations, there is still some question as to whether these explanations do in fact reflect reality. Most observations cannot adequately address whether the ESWs were generated in situ because the particle measurements do not have the time resolution necessary to identify phase space holes on such short time scales. Full distribution functions need to be made on the order of $1 \mathrm{~ms}$ and much less in order to see holes in phase space, for example. The current time resolution for obtaining distribution functions of most particle instruments is far greater than this, often a few to several seconds. Regarding models and simulations, they have the potential to provide tremendous insight into ESW generation, but assumptions and initial and boundary conditions which do not adequately reflect the space conditions, especially in light of the dynamical nature of space, are often involved. Thus, there is the need to connect the models/simulations and space observations with laboratory experiments as described in Sect. 3.

Regardless of the difficulties in coming to a final determination as to how the nonlinear ESWs in space are generated since instabilities are known to occur over a very short period of time, we can discuss some of the possibilities for the super-substorm event. The observed field-aligned electron fluxes, sometimes counter streaming, on Cluster could certainly lead to the local generation of the ESWs through a beam or counter-streaming instability. In addition, the kinetic Buneman instability is considered a viable generation mechanism for the super-substorm ESWs based on a possible interaction between the ions and electrons. This instability arises when the ion beam interacts with an electron beam to produce a wave moving at nearly the ion velocity. In a scenario related to a large-scale potential ramp, these slow waves saturate by trapping both electrons and ions, leading to a train of alternating electron and ion phase space holes moving at a slightly slower velocity than the accelerated ions (Goldman et al., 2003). Even though the predicted slow phase-space holes cannot account for the electron-scale
ESWs observed in the auroral bulge phase and in the LAPD, the Buneman instability in general may be relevant to longer time-duration ESWs. Unfortunately, we cannot determine the speed of these ESWs for comparison to the speed of the accelerated ions in order to conclude whether the kinetic Buneman instability is indeed active. Further, for both beam and Buneman instabilities where the ESWs would be identified as electron phase space holes, we cannot verify their presence due to the insufficient time resolution of the electron distribution functions. The generation of the ESWs detected on Geotail in the far magnetotail were also attributed to an electrostatic beam instability (Omura et al., 1996). Interestingly, in simulations carried out by Goldman et al. (1999) creating a two-stream electron instability, ESWs are produced, as well as whistler mode waves well after instability onset. Cluster, as noted in section $2 b$, observes whistler mode waves frequently in the same time period that the ESWs are observed, which could be one of the byproducts of the electron trapping/ESW generation mechanism.

Another explanation for the local generation of the ESWs lends itself to these observations due to the very dynamical nature of the event. It is similar to that proposed by Lakhina et al. (2004) and Tsurutani et al. (2003) using data from the Polar spacecraft for a magnetic hole event in the polar cap boundary layer. It relies on low frequency waves (Alfvén and/or obliquely propagating proton cyclotron waves) to provide the free energy for electron heating which produces electron currents to drive some much higher frequency instabilities, namely the lower-hybrid, ion acoustic, bi-stream or counter-streaming electron, and electrostatic ion cyclotron instabilities. These instabilities will saturate in about 100400 electron plasma periods by trapping electrons, which, in turn, leads to generation of electron phase space holes. In light of the short time duration of the observed ESWs, the electron counter-streaming or beam instabilities are the most plausible candidates for generating the ESWs. For the supersubstorm case discussed in this paper, it would appear that all of the elements necessary to initiate and excite the processes and instabilities that create phase space holes are present locally, i.e., Alfvén and ion cyclotron waves, electron heating and currents, and field-aligned electron fluxes.

Finally, there exists the possibility for the electron acoustic instability to lead to the generation of the ESWs. As discussed in Ghosh et al. (2008) for three magnetospheric regions (bow shock, magnetosheath, cusp), the requirement is that energetic multi-ion species be present along with fieldaligned fluxes or beams of electrons. We have shown that both hydrogen and oxygen ions are present for this event and that the oxygen ions are a substantial part of the ion density, along with hydrogen, which is not usually the case in this region. Before electrostatic broadband noise was known to consist of isolated ESWs, Ashour-Abdalla and Okuda (1986) had concluded that the electron acoustic instability could be excited in the magnetotail. The electron acoustic instability will lead to enhancements (Berthomier et al., 2000) or 
decreases in electron density, as opposed to holes in electron phase space. Recently, Lakhina et al. (2008b) have developed a model to study large amplitude ion-acoustic and electronacoustic waves in an unmagnetized multi-component plasma system consisting of cold background electrons and ions, a hot electron beam and a hot ion beam. For typical parameters of the plasma sheet boundary layer (Grabbe and Eastman, 1984), the model predicted positive potential slow ionacoustic, ion-acoustic and electron-acoustic solitons. It is interesting to note that the electron-acoustic solitons have maximum electric field amplitudes of $E_{\max }=(4-10) \mathrm{mV} / \mathrm{m}$, widths $\sim(7-10) \mathrm{km}$, and Mach number of 53.4 (in terms of hot ion thermal speed). In terms of electron parameters, the electron-acoustic soliton velocities and widths scale as $\sim 1.24 v_{T e}$ and $\sim 30 \lambda_{D e}$, respectively. Thus, the predicted electron-acoustic solitons have velocities very close to the velocity of the ESWs observed in the LAPD experiment. However, the scale size of the electron-acoustic solitons is about a factor of 4 larger than the LAPD ESW scale size. With regard to the ion acoustic instability, we find it highly unlikely that the ESWs are ion acoustic solitons based purely on the observation that the space ESWs have time duration scales consistent with electron time scales.

In closing our discussion of the generation mechanism for the space observations, we note the possibility that the ESWs were generated elsewhere and propagated to the Cluster spacecraft. It has been established through various published ESW studies that ESWs do propagate, often along magnetic field lines. It is not possible through the Cluster data set to determine whether the ESWs have propagated to the spacecraft, whether over small or large distances. Thus, our analysis was restricted to discussing those generation mechanisms that could create the ESWs locally based on the Cluster observations.

With regard to generation of the ESWs in the LAPD experiments, as noted in Sect. 3 we have ruled out the electronelectron two stream instability as the generation mechanism because the two-stream generated ESWs are expected to propagate at about half the beam speed whereas the ESW velocity is substantially smaller than the beam speed. Furthermore, the conditions for the classical Buneman instability to develop are not present because at least $90 \%$ of the electrons are at rest with respect to the ions (the beam density is less than $10 \%$ of the background density). Since the results of these experiments are still being analyzed, it is not possible at present to conclude which generation mechanism is most likely active in the LAPD.

\section{Conclusions}

The Cluster spacecraft, which were located about $18.8 R_{E}$ down the Earth's magnetotail on 24 August 2005 and moving northward from the inner plasma sheet to the lobes, observed many interesting features associated with the onset of a super-substorm event around 09:42 UT. The primary result being reported here, and one which has not been previously reported in the literature, is the appearance of numerous nonlinear Electrostatic Solitary Waves in the magnetotail in association with a super-substorm onset. These ESWs are somewhat more numerous, of shorter duration, and of larger amplitude than is usually encountered at this distance down the tail by Cluster.

Clearly, the ESWs observed in the period 09:48-09:53 by WBD on at least three of the Cluster spacecraft are associated with super-substorm onset and the ensuing response of the magnetosphere. The electrons appear to be heated early on and then develop field-aligned fluxes during the interval in which the most ESWs are observed. EDI observes the ensuing electron currents. During the same ESW interval, ions are streaming tailward up to several hundred $\mathrm{km} / \mathrm{s}$. In addition the makeup of the ions at Cluster's location indicates that a significant constituent is $\mathrm{O}+$, only slightly less than the dominant $\mathrm{H}+$. Alfvén waves and low frequency ion cyclotron waves are present, as well as whistler waves of a few hundred $\mathrm{Hz}$. Thus, we have a very dynamical situation where it is not surprising that ESWs are seen in abundance since they are almost always found in boundary/current layers and turbulent plasmas.

We have shown that ESWs can be generated in the laboratory using an electron beam. The ESWs generated in the laboratory thus far have much larger amplitudes, but their time durations are slightly larger than the electron plasma period, just as for the ESWs observed during this super-substorm event. In the future we hope to connect the laboratory observations to those in space through PIC code simulations.

We suggested plausible methods for the generation of these solitary waves through beam, counter-streaming and kinetic Buneman type instabilities based on previous observations, theory, and simulations. These generation mechanisms result in BGK type electron phase space holes and electron density enhancements and decreases which would be represented in AC electric field waveforms as pulses (the ESWs). It is beyond the scope of this paper to test the various generation methods discussed above. Based purely on the observational considerations, particularly the electron-scale time durations of the ESWs, however, electron-type instabilities are considered the most probable generation mechanisms during the time of the 24 August 2005 super-substorm. Generation mechanisms involving the observed two ion species are considered less probable since the velocity and inertia differences between the two species is irrelevant from the point of view of the electrons and the ESWs have time scales more consistent with those of electrons.

Acknowledgements. We thank the reviewers for their helpful comments which have been taken into consideration in our final manuscript. We also thank the organizers of the Nonlinear Wave Workshop held in Beaulieu, France in April 2008 and of the Alfvén Workshop held in Warsaw, Poland in September 2007 for inviting us to present this work. JSP acknowledges the support of NASA 
Goddard Space Flight Center under Grant NNX07AI24G and the U.S. Department of Energy under Grant DE-FG02-07ER54943. JSP would also like to acknowledge the NASA Deep Space Network schedulers, engineers and operators for obtaining the Cluster WBD data discussed here and Julie Dowell and Joanne Seeberger for processing it, as well as the ESA Operations Centre, the Cluster Joint Science Operations Centre at Rutherford Appleton Laboratory, and Sheffield University who were instrumental in obtaining the Cluster data discussed here, including that of WBD. Finally, JSP acknowledges the crucial support provided by NASA's SSCWeb for operations planning and data analysis purposes. The research at UNH was supported by DOE grants DE-FG02-07ER54941 and DE-FG02-06ER54894. Portions of this research were performed at the Jet Propulsion Laboratory, California Institute of Technology under contract with NASA. OS acknowledges additional support from the NSF award No. 0307319/ME 842 and GAAV grant IAA 301120601. SG acknowledges support from the STFC Rolling Grant at MSSL. The STAFF team and BG acknowledge support from CNES through various grants. GSL thanks the Indian National Science Academy, New Delhi for the support under the Senior Scientist Scheme. We gratefully acknowledge support of Walter Gekelman, Steve Vincena, Pat Pribyl and Franklin Chiang at the LAPD for assisting us with the laboratory measurements. The experiments done at the LAPD device are funded by the Basic Plasma Facility, a user facility funded by the National Science Foundation and the Department of Energy.

Edited by: R. Pottelette

Reviewed by: two anonymous referees

\section{References}

Akasofu, S.-I.: The development of the auroral substorm, Planet. Space Sci., 12, 273-182, 1964.

Ashour-Abdalla, M. and Okuda, H.: Electron-acoustic instabilities in the geomagnetic tail, Geophys. Res. Lett., 13, 366-369, 1986.

Balogh, A., Dunlop, M. W., Cowley, S. W., Southwood, D. J., Thomlinson, J., et al.: The Cluster Magnetic Field Experiment, Space Sci. Rev., 79, 65-91, 1997.

Bernstein I. B., Greene J. M., and Kruskal M. D.: Exact Nonlinear Plasma Oscillations, Phys. Rev., 108, 546-550, 1957.

Berthomier, M., Pottelette, R., Malingre, M., and Khotyaintsev, Y.: Electron-acoustic solitons in an electron-beam plasma system, Phys. Plasmas., 7, 2987-2994, 2000.

Cattell, C. A., Dombeck, J., Wygant, J. R., Hudson, M. K., Mozer, F. S., et al.: Comparisons of Polar satellite observations of solitary wave velocities in the plasma sheet boundary and the high altitude cusp to those in the auroral zone, Geophys Res. Lett., 26, 425-428, 1999.

Cattell, C., Dombeck, J., Wygant, J., Drake, J. F., Swisdak, M., et al.: Cluster observations of electron holes in association with magnetotail reconnection and comparison to simulations, J. Geophys. Res., 110, A01211, doi:10.1029/2004JA010519, 2005.

Chen, L.-J., Thouless, D. J., and Tang, J.-M.: Bernstein-GreeneKruskal solitary waves in three-dimensional magnetized plasma, Phys. Rev. E, 69, 055402(R), 2004.

Chen, L.-J, Pickett, J., Kintner, P., Franz, J., and Gurnett, D.: On the width-amplitude inequality of electron phase space holes, J. Geophys. Res., 110, A09211, doi:10.1029/2005JA011087, 2005.
Cornilleau-Wehrlin, N., Chanteur, G., Perraut, S., Rezeau, L., Robert, P., Roux, A., de Villedary, C., Canu, P., Maksimovic, M., de Conchy, Y., Hubert, D., Lacombe, C., Lefeuvre, F., Parrot, M., Pinçon, J. L., Décréau, P. M. E., Harvey, C. C., Louarn, Ph., Santolik, O., Alleyne, H. St. C., Roth, M., Chust, T., Le Contel, O., and STAFF team: First results obtained by the Cluster STAFF experiment, Ann. Geophys., 21, 437-456, 2003, http://www.ann-geophys.net/21/437/2003/.

Décréau, P. M. E., Fergeau, P., Krannosels'kikh, V., Lévêque, M., Martin, Ph., et al.: Whisper, A resonance sounder and wave analyzer: performances and perspectives for the Cluster mission, Space Sci. Rev., 79, 157-193, 1997.

Deng, X. H., Tang, R. X., Matsumoto, H., Pickett, J. S., Fazakerley, A., et al.: Observations of electrostatic solitary waves associated with reconnection by Geotail and Cluster, Adv. Space Res., 37, 1373-1381, doi:10.1016/j.asr.2005.05.129, 2006.

Drake, J. F., Swisdak, M., Cattell, C., Shay, M. A., Rogers, B. N., and Zeiler, A.: Formation of electron holes and particle energization during magnetic reconnection, Science, 299, 873-877, 2003.

Dubouloz, N., Pottelette, R., Malingre, M., and Treumann, R. A.: Generation of Broadband Electrostatic Acoustic Solitons, Geophys. Res. Lett., 18, 155-158, 1991.

Franz, J. R., Kintner, P. M., and Pickett, J. S.: POLAR observations of coherent electric field structures, Geophys. Res. Lett., 25, 1277-1280, 1998.

Franz, J. R., Kintner, P. M., Seyler, C. E., Pickett, J. S., and Scudder, J. D.: On the perpendicular scale of electron phase-space holes, Geophys. Res. Lett., 27, 169-172, 2000.

Franz, J. R., Kintner, P. M., Pickett, J. S., and Chen, L.-J.: Properties of small amplitude electron phase-space holes observed by Polar, J. Geophys. Res., 110, A09212, doi:10.1029/2005JA011095, 2005.

Ghosh, S. S., Pickett, J. S., Lakhina, G. S., Winningham, J. D., Lavraud, B., and Décréau, P. M. E.: Parametric analysis of positive amplitude electron acoustic solitary waves in a magnetized plasma and its application to boundary layers, J. Geophys. Res., 113, A06218, doi:10.1029/2007JA012768, 2008.

Goldman, M. V., Oppenheim, M. M., and Newman, D. L.: Nonlinear two-stream instability as an explanation for auroral bipolar wave signatures, Geophys. Res. Lett., 26, 1821-1824, 1999.

Goldman, M. V., Newman, D. L., and Ergun, R. E.: Phase-space holes due to electron and ion beams accelerated by a currentdriven potential ramp, Nonlin. Processes Geophys., 10, 37-44, 2003, http://www.nonlin-processes-geophys.net/10/37/2003/.

Grabbe, C. L. and Eastman, T. E.: Generation of broadband electrostatic noise by ion beam instabilities in the magnetotail, J. Geophys. Res., 89, 3865-3872, 1984.

Gurnett, D. A., Huff, R. L., and Kirchner, D. L.: The Wide-Band Plasma Wave Investigation, Space Sci. Rev., 79, 195-208, 1997.

Gustafsson, R., Bostrom, R., Holback, B., Holmgren, G., Lundgren, A., et al.: The Electric Field and Wave Experiment for the Cluster mission, Space Sci. Rev., 79, 137-156, 1997.

Johnstone, A. D., Alsop, C., Carter, P. J., Coates, A. J., Coker, A. J., et al.: PEACE: A plasma electron and current experiment, Space Sci. Rev., 79, 351-398, 1997.

Kakad, A. P., Singh, S. V., Reddy, R. V., Lakhina, G. S., Tagare, S. G., and Verheest, F.: Generation mechanism for 
electron acoustic solitary waves, Phys. Plasmas., 14, 052305, doi:10.1063/1.2732176, 2007.

Lakhina, G. S., Tsurutani, B. T., and Pickett, J.: Association of Alfvén waves and proton cyclotron waves with electrostatic bipolar pulses: magnetic hole events observed by Polar, Nonlin. Processes Geophys., 11, 205-213, 2004, http://www.nonlin-processes-geophys.net/11/205/2004/.

Lakhina, G. S., Kakad, A. P., Singh, S. V., and Verheest, F.: Ionand electron-acoustic solitons in two-electron temperature space plasmas, Phys. Plasmas., 15, 062903, doi:10.1063/1.2930469, 2008a.

Lakhina, G. S., Singh, S. V., Kakad, A. P., Verheest, F., and Bharuthram, R.: Study of nonlinear ion- and electron-acoustic waves in multi-component space plasmas, Nonlin. Processes Geophys., 15, 903-913, 2008b, http://www.nonlin-processes-geophys.net/15/903/2008/.

Lefebvre, B., Chen, L.-J., Gekelman, W., Kintner, P., Pickett, J., Pribyl, P., Vincena, S., Chiang, F., and Judy, J.: Electrostatic solitary waves generated by injecting suprathermal electron beams in laboratory plasmas, Phys. Rev. Lett., in preparation, 2009.

Matsumoto, H., Kojima, H., Miyatake, T., Omura, Y., Okada, M., et al.: Electrostatic solitary waves (ESW) in the magnetotail: BEN wave forms observed by GEOTAIL, Geophys. Res. Lett., 21, 2915-2918, 1994.

Matsumoto, H., Deng, X. H., Kojima, H., and Anderson, R. R.: Observation of Electrostatic Solitary Waves associated with reconnection on the dayside magnetopause boundary, Geophys. Res. Lett., 30, 1326, doi:10.1029/2002GL016319, 2003.

Omura, Y., Matsumoto, H., Miyake, T., and Kojima, H.: Electron beam instabilities as generation mechanism of electrostatic solitary waves in the magnetotail, J. Geophys. Res., 101, 2685-2697, 1996.

Paschmann, G., Melzner, F., Frenzel, R., Vaith, H., Parigger, P., et al.: The Electron Drift Instrument for Cluster, Space Sci. Rev., 79, 233-269, 1997.

Pickett, J. S., Chen, L.-J., Kahler, S. W., Santolík, O., Gurnett, D. A., Tsurutani, B. T., and Balogh, A.: Isolated electrostatic structures observed throughout the Cluster orbit: relationship to magnetic field strength, Ann. Geophys., 22, 2515-2523, 2004, http://www.ann-geophys.net/22/2515/2004/.

Pickett, J. S., Chen, L.-J., Kahler, S. W., Santolík, O., Goldstein, M. L., Lavraud, B., Décréau, P. M. E., Kessel, R., Lucek, E., Lakhina, G. S., Tsurutani, B. T., Gurnett, D. A., CornilleauWehrlin, N., Fazakerley, A., Rème, H., and Balogh, A.: On the generation of solitary waves observed by Cluster in the nearEarth magnetosheath, Nonlin. Processes Geophys., 12, 181-193, 2005 http://www.nonlin-processes-geophys.net/12/181/2005/.

Pickett, J. S., Chen, L.-J., Mutel, R. L., Christopher, I. W., Santolík, O., et al., Furthering our understanding of electrostatic solitary waves through Cluster multispacecraft observations and theory, Adv. Space Res., 41, 1666-1676, 2008.
Pottelette, R. and Treumann, R. A.: Impulsive broadband electrostatic noise in the cleft: A signature of dayside reconnection, J. Geophys. Res., 103, 9299-9307, 1998.

Rème, H., Aoustin, C., Bosqued, J. M., Dandouras, I., Lavraud, B., Sauvaud, J. A., Barthe, A., Bouyssou, J., Camus, Th., CoeurJoly, O., Cros, A., Cuvilo, J., Ducay, F., Garbarowitz, Y., Medale, J. L., Penou, E., Perrier, H., Romefort, D., Rouzaud, J., Vallat, C., Alcaydé, D., Jacquey, C., Mazelle, C., d’Uston, C., Möbius, E., Kistler, L. M., Crocker, K., Granoff, M., Mouikis, C., Popecki, M., Vosbury, M., Klecker, B., Hovestadt, D., Kucharek, H., Kuenneth, E., Paschmann, G., Scholer, M., Sckopke (†), N., Seidenschwang, E., Carlson, C. W., Curtis, D. W., Ingraham, C., Lin, R. P., McFadden, J. P., Parks, G. K., Phan, T., Formisano, V., Amata, E., Bavassano-Cattaneo, M. B., Baldetti, P., Bruno, R., Chionchio, G., Di Lellis, A., Marcucci, M. F., Pallocchia, G., Korth, A., Daly, P. W., Graeve, B., Rosenbauer, H., Vasyliunas, V., McCarthy, M., Wilber, M., Eliasson, L., Lundin, R., Olsen, S., Shelley, E. G., Fuselier, S., Ghielmetti, A. G., Lennartsson, W., Escoubet, C. P., Balsiger, H., Friedel, R., Cao, J.-B., Kovrazhkin, R. A., Papamastorakis, I., Pellat, R., Scudder, J., and Sonnerup, B.: First multispacecraft ion measurements in and near the Earth's magnetosphere with the identical Cluster ion spectrometry (CIS) experiment, Ann. Geophys., 19, 1303-1354, 2001 , http://www.ann-geophys.net/19/1303/2001/.

Singh, S. V. and Lakhina, G. S.: Generation of electron-acoustic waves in the magnetosphere, Planet. Space Sci., 49, 107-114, 2001.

Singh, S. V., Reddy, R. V., and Lakhina, G. S.: Broadband electrostatic noise due to nonlinear electron-acoustic waves, Adv. Space Res. 28, 1643-1648, 2001.

Sonnerup, B. U. O. and Cahill Jr., L. J.: Magnetopause structure and attitude for Explorer 12 observations, J. Geophys. Res., 72, 171-183, 1967.

Temerin, M., Cerny, K., Lotko, W., and Mozer, F. S.: Observations of double layers and solitary waves in the auroral plasma, Phys. Rev. Lett., 48, 1175-1179, 1982.

Tsurutani, B. T. and Zhou, X.-Y.: Interplanetary shock triggering of substorms: Wind and Polar, Adv. Space Res., 31, 1063-1067, 2003.

Tsurutani, B. T., Dasgupta, B., Arballo, J. K., Lakhina, G. S., and Pickett, J. S.: Magnetic field turbulence, electron heating, magnetic holes, proton cyclotron waves, and the onsets of bipolar pulse (electron hole) events: a possible unifying scenario, Nonlin. Processes Geophys., 10, 27-35, 2003 , http://www.nonlin-processes-geophys.net/10/27/2003/.

Zhou, X.-Y. and Tsurutani, B. T.: Interplanetary shock triggering of nightside geomagnetic activity: Substorms, pseudobreakups and quiescent events, J. Geophys. Res., 106, 18957-18967, 2001 